\title{
brain-coX: investigating and visualising gene co-expression in seven human brain transcriptomic datasets
}

\author{
Saskia Freytag ${ }^{1,2^{*}}$, Rosemary Burgess ${ }^{3}$, Karen L. Oliver $^{1,3}$ and Melanie Bahlo ${ }^{1,2,4}$
}

\begin{abstract}
Background: The pathogenesis of neurological and mental health disorders often involves multiple genes, complex interactions, as well as brain- and development-specific biological mechanisms. These characteristics make identification of disease genes for such disorders challenging, as conventional prioritisation tools are not specifically tailored to deal with the complexity of the human brain. Thus, we developed a novel webapplication-brain-coX - that offers gene prioritisation with accompanying visualisations based on seven gene expression datasets in the post-mortem human brain, the largest such resource ever assembled.

Results: We tested whether our tool can correctly prioritise known genes from 37 brain-specific KEGG pathways and 17 psychiatric conditions. We achieved average sensitivity of nearly $50 \%$, at the same time reaching a specificity of approximately $75 \%$. We also compared brain-coX's performance to that of its main competitors, Endeavour and ToppGene, focusing on the ability to discover novel associations. Using a subset of the curated SFARI autism gene collection we show that brain-coX's prioritisations are most similar to SFARI's own curated gene classifications.
\end{abstract}

Conclusions: brain-coX is the first prioritisation and visualisation web-tool targeted to the human brain and can be freely accessed via http://shiny.bioinf.wehi.edu.au/freytag.s/.

\section{Background}

The World Health Organization estimates that around 450 million people worldwide suffer from mental or neurological conditions, placing these disorders at the top of the list of global disease burdens [1]. A better understanding of biochemical and morphological abnormalities in affected brains can help alleviate this burden. Next to non-invasive neuroimaging and post-mortem histological analysis, the identification of genes involved in the pathogenesis of these disorders is the most promising avenue to improve our knowledge and consequently develop better diagnostics, treatments and targeted therapeutics [2].

In recent years, genome-wide association studies, as well as high-throughput sequencing of families, have identified hundreds of variants located in, or near,

\footnotetext{
* Correspondence: freytag.s@wehi.edu.au

${ }^{1}$ Population Health and Immunity Divison, The Walter and Eliza Hall Institute

of Medical Research, $1 \mathrm{G}$ Royale Parade, 3052 Parkville, Australia

${ }^{2}$ Department of Medical Biology, University of Melbourne, 1G Royale Parade, 3052 Parkville, Australia

Full list of author information is available at the end of the article
}

coding regions compellingly statistically associated with mental and neurological disorders [3, 4]. For many of the variants, however, the functional alleles and mechanisms that give rise or contribute to these disorders remain elusive. For example, while more than 100 loci are associated with schizophrenia, few genes have been implicated in the biological process underlying this genetically complex disease [5]. Similarly, about 1000 copy number variants and rare and common variants have been found to be associated with autism [6], but little is known about how they confer risk.

Designing and performing scientific experiments to generate functional evidence for the involvement of a gene in a mental or neurological disorder is often challenging. The cost of such experiments is typically high, in particular when human brain tissue is necessary. Furthermore, because of the large number of putative disease genes only a subset of genes can be followed up. Fortunately, computational methods as well as visualisation techniques exist that can help to prioritise which candidate genes to pursue with such methods. These 
methods are referred to as in silico prioritisation. These methods typically rely on knowledge collected in genomic databases, such as the Online Mendelian Inhertiance in Man (OMIM) database [7], as well as gene expression data from healthy individuals $[8,9]$. Popular examples of computational methods offering in silico prioritisation include Endeavour [10] and ToppGene [11]. One of the most frequently employed visualisation tools for gene networks is String [12].

Both in silico prioritisation and gene network visualisation tools have been successfully applied to many diseases [13, 14]. Nevertheless, most tools are biased towards what is already known due to their reliance on genomic databases and literature searches via databases such as PubMed [15]. Because of this known bias some tools also integrate gene expression data from healthy individuals to implicate disease pathways discovered de novo from the data. However, such gene expression data are usually generated from easily obtained sources, such as blood or lymphocytes, and thus may not reflect the pathways in the relevant disease tissue $[16,17]$. Gene expression in the brain is uniquely different from other tissues, reflecting the complex biological processes in the brain [18]. Leveraging such brain-specific gene signatures has indeed been shown to be beneficial in uncovering disease genes for epileptic encephalopathies [19]. Furthermore, many available tools do not take into account that gene expression varies considerably over the course of an individual's development, especially in the brain. For example, in the human brain, Kang et al. [20] observed that gene expression is regulated to a large degree temporally and only to a lesser extent spatially.

Very few tools offer both in silico prioritisation and gene network visualisation, which hinders interpretation and design of functional downstream analysis [8] (one notable exception is the downloadable application NETBAG [21]). brain-coX is a novel web-application focusing on gene prioritisation and exploration of gene networks for diseases that originate in human brain tissue. Unlike any of the existing tools, brain-coX's prioritisations are based solely on brain expression data, making use of up to seven available large datasets measuring gene expression in the developing and ageing human brain. These datasets were processed and cleaned in a homogeneous manner, ensuring maximal reproducibility of results across datasets. To our knowledge this is the first time results from these seven precious brain expression datasets are directly comparable, within one resource. Besides prioritisations, brain-coX also allows users to investigate pathway membership and to explore changes in gene networks throughout brain development via interactive visualisations. Such temporal changes in gene networks have been hypothesized to play a key role in many neurological and mental disorders, with many such disorders showing distinct ages of onset. Finally, we designed brain-coX to be user-friendly and easily accessible through a website to facilitate use by researchers who are not comfortable with command line tools.

\section{Implementation \\ Datasets \\ Dataset descriptions}

We downloaded seven published and publicly available datasets of gene expression from post-mortem human brain tissue samples (Table 1). For six out of seven datasets, samples were collected from individuals deemed to be normal with respect to mental and neurological disorders. The Hernandez et al. dataset [22] contains some individuals with unknown disease status. Datasets differ widely with regards to age range of individuals, number of individuals and number of samples as well as tissue types collected from each brain. To cater for this, braincoX allows the user to select any combination of these datasets. Furthermore, users are able to further subset data by specifying developmental periods of interest. To this end, the individuals contributing samples were assigned to 15 different developmental periods according to their age at death (Table 2). This option facilitates targeted prioritisation and gene exploration for specific diseases. An example would be a disease with onset in childhood where a focus on brain samples from this time period are likely to be much more informative than samples from other time periods.

Table 1 Key features of the seven different gene expression datasets of the developing and ageing brains

\begin{tabular}{lllll}
\hline Gene expression resource/publication & Platform & Number of individuals & Average number of arrays per brain & Number of time periods \\
\hline Hawrylycz et al. [31] & Agilent & 10 & 406 & 2 \\
Miller et al. [44] & Agilent & 4 & 328 & 2 \\
Colantuoni et al. [45] & Custom & 266 & 1 & 11 \\
Kang et al. [20] & Affymetrix & 57 & 24 & 15 \\
Hernandez et al. ${ }^{\text {a }}$ [22] & Illumina & 397 & 2 & 8 \\
Trabzuni et al. [46] & Affymetrix & 134 & 9 & 4 \\
Zhang et al. [47] & Agilent & 101 & 3 & 3
\end{tabular}

${ }^{a}$ This dataset contains some individuals who were not normal with respect to neurological and mental health disorders 
Table 2 Fifteen developmental periods of the human brain as defined by Kang et al.

\begin{tabular}{lll}
\hline Period & Description & Age range \\
\hline 1 & Embryonic & $4-8 \mathrm{PCW}$ \\
2 & Early fetal & $8-10 \mathrm{PCW}$ \\
3 & Early fetal & $10-13 \mathrm{PCW}$ \\
4 & Early mid-fetal & $13-16 \mathrm{PCW}$ \\
5 & Early mid-fetal & $16-19 \mathrm{PCW}$ \\
6 & Late mid-fetal & $19-24 \mathrm{PCW}$ \\
7 & Late fetal & $24-18 \mathrm{PCW}$ \\
8 & Neonatal and early infancy & Birth to $6 \mathrm{M}$ \\
9 & Late infancy & $6 \mathrm{M}-1 \mathrm{Y}$ \\
10 & Early childhood & $1-6 \mathrm{Y}$ \\
11 & Middle and late childhood & $6-12 \mathrm{Y}$ \\
12 & Adolescence & $12-20 \mathrm{Y}$ \\
13 & Young adulthood & $20-40 \mathrm{Y}$ \\
14 & Middle adulthood & $40-60 \mathrm{Y}$ \\
15 & Late adulthood & $60+\mathrm{Y}$ \\
\hline
\end{tabular}

$M$ months, $P C W$ post-conception weeks, $Y$ years

\section{Pre-processing and data cleaning}

Different experimental protocols and microarray platforms were used in the generation of these seven datasets, leading to diverse sources of unwanted biological and technical variation. Thus, homogeneous preprocessing and data cleaning are vital in order to ensure that these heterogeneous datasets are comparable [23]. During pre-processing each sample was assessed for its quality and samples with poor quality spot plots, unusual plots of log-intensity ratios versus log-intensity averages or abnormal gene expression distributions were excluded. After pre-processing, each dataset is treated separately with one of two implemented cleaning strategies. Users can choose between conventional background correction [24] in combination with quantile normalisation [25] or removal of unwanted variation (RUV) [26], a data-driven approach. Unlike most other cleaning approaches, such as ComBat [27], these two approaches do not require meta data (i.e. batches, laboratory, etc.) on the samples, which in most datasets was partially or not at all available.

RUV removes unwanted variation in an adaptive manner with the help of negative control genes. Such genes are affected by unwanted variation, but crucially not by the biological variation of interest. The default setting in brain-coX is to take all house-keeping genes as negative control genes, but these can also be empirically chosen. When unwanted variation and biological variation of interest are correlated with each other, RUV removes biological signal. In order to account for such correlation to a degree, brain-coX applies a version of RUV with a regularization parameter, as previously described [28]. To prevent further removal of biological variation of interest, brain-coX also excludes known disease genes, candidates and further genes specified by the user from being negative control genes. This method has been demonstrated to reliably recover gene-gene correlations, which form the basis of in silico prioritisation and network visualisations. Furthermore, its application to a subset of the brain datasets demonstrated improved reproducibility across datasets compared to other cleaning strategies [28]. Here, we also demonstrate increased accuracy prioritising known pathway genes compared to background correction and quantile normalisation (Additional file 1: Figures S1 and S2). Furthermore, RUV also considerably reduces differences between datasets. When the seven datasets are combined, differences between the datasets are noticeably reduced and the remaining clustering can be attributed to developmental differences rather than data sources (compare Additional file 1: Figures S3, S4, S5 and S6).

\section{Prioritisation}

brain-coX prioritises user-supplied candidate genes via the guilt-by-association principle [29]. This principle assumes that the most promising candidate genes will be the ones that are associated with genes already known to be involved in the disease. Such candidates are likely to be part of the same biological network(s) that, when disrupted, lead to the development of the disease. The reliance on this principle means that the user is required to supply already known disease genes in order to prioritise their candidate genes. This approach has been shown to work well in many neurodevelopmental disorders where the list of discovered genes continues to grow. However, it should be noted that the guilt-by-association principle assumes that all disease genes fall into a small number of convergent pathways. If this is not the case, discovery of new disease genes is unlikely.

As gene prioritisation is the focus of brain-coX, we implemented an improved version of a prioritisation strategy, BrainGEP, proposed by Oliver et al. [30]. Using a retrospective analysis, they were able to assess the validity of their prioritisations. In Oliver et al. 179 putative epileptic encephalopathy candidates were examined, of which 19 had been prioritised in 2013. They found that six candidates had since been confirmed, of which their prioritisation had predicted five [19]. This result is based on the use of only the Allen Human Brain Atlas expression dataset [31] while brain-coX prioritises candidates on up to seven datasets simultaneously and compares the results. Furthermore, brain-coX employs a different weighting of the simple Pearson correlation to BrainGEP. In brain-coX, correlations are weighted by the inverse of the number of samples contributed by the 
respective donor in order to take into account dependencies.

The prioritisation can be summarised in three steps, which are conducted on each of the seven datasets available in brain-coX separately (also compare Additional file 1: Figure S7).

\section{Step 1: Determination of background correlation}

A random set of genes, the size of the candidate gene set, is selected. The absolute weighted correlation between these random genes and the known disease genes is then calculated. For each gene only the maximum absolute correlation is retained. This step is repeated 1000 times.

\section{Step 2: Determination of correlation threshold}

The user selects a proportion of non-disease genes allowed to be prioritised. The 1000 repeats of step 1 can then be used to determine the threshold for the absolute correlation, ensuring the user-selected proportion of random genes gets prioritised on average. Note that this proportion is an overestimate as not all randomly selected genes will be truly biologically independent of the disease genes.

\section{Step 3: Prioritisation}

Finally, brain-coX calculates the correlation between the candidate genes and the disease genes. With the cutoff for the absolute correlation established, candidate genes that have a maximum absolute correlation with any disease gene greater than the cutoff are prioritised.

As prioritisation is performed separately in every selected dataset, the number of datasets a candidate gene is prioritised in can thereby be seen as an indicator of the likelihood that the candidate gene is truly associated with the disease genes. Candidate genes can be further ranked by their sum of all absolute correlations, above the calculated threshold with any known disease gene.

\section{Exploration through visualisation Network visualisation}

brain-coX has extensive visualisation options that allow an intuitive understanding of the prioritisation results. The tool offers two types of network visualisations. The first uses the datasets separately and is designed to highlight the effect of individual datasets on prioritisation results. The user can also interactively assess how different choices of parameters, such as the proportion threshold, influence the results. For the second visualisation, datasets were combined and the user can investigate different clustering algorithms on the gene-gene correlation heatmap. Furthermore, users also have the option to explore partial correlations, which control for indirect interactions between genes.

\section{Investigating the effect of brain development}

Gene expression networks are known to alter in response to environmental cues and factors during development [32]. Users can explore such changes with heatmaps of gene-gene correlations estimated for each time period independently. They can also focus on the changes occurring in gene regulation in the normal human brain between sets of time periods. We believe that this feature in particular may help to pinpoint diseaserelevant developmental mechanisms that are disrupted in patients. We have included a case study in Additional file 1 to explain the use of these features in learning more about candidate genes.

\section{Interface}

The graphical user interface of brain-coX was built using shiny [33]. Like other shiny applications, brain-coX leverages R [34] and Bioconductor [35] resources for the underlying calculations and plot output. Due to shiny's inherent reactive programming framework, output is only updated when the user changes the settings or instigates a new query. There are two ways to run braincoX: firstly, it can be accessed through our web-server (http://shiny.bioinf.wehi.edu.au/freytag.s/), requiring no further programmes to be installed; secondly, it is available as a local version once the software is downloaded and installed. The latter has the advantage of reducing computational time and not being limited by the host web-server's current load. However, this requires a recent version of $\mathrm{R}$ and several additional $\mathrm{R}$ and Bioconductor packages on which several steps rely. Furthermore, executing the application requires basic knowledge of $\mathrm{R}$.

For a detailed example explaining the use of brain-coX to identify zinc transporter genes that may play a role in febrile seizures see Additional file 1 and Additional files 2, 3 and 4 for the associated data.

\section{Results}

\section{Statistical benchmarking}

We followed the leave-one-out cross-validation approach described by Aerts et al. [10]. In this approach one gene is deleted from the known set of genes and termed the "defector" gene. The ability to prioritise this gene in a list of 99 other candidates, made up of random genes not known to be associated, determines the accuracy. Unlike Aerts et al., we used two different types of known gene sets that will reflect a spectrum of networks, with some gene sets likely to be connected within one network and other gene sets showing very little connection. The former gene sets will do well with our approach, the latter will not. The first set of genes consists of 37 KEGG pathways [36] which function in the human brain as judged by keywords search (Additional file 1: 
Table S2). The second set of genes was automatically mined from the PsyGeNet database [37], a resource that stores genes associated with psychiatric diseases (for a full list see Additional file 1: Table S3). This set contained 17 diseases, such as major affective disorder and anhedonia, and their known genes.

For the prioritisation approach implemented in braincoX with RUV normalisation, we also examined the effect of using multiple datasets. To do this we defined a successful prioritisation as a gene prioritised in at least $\mathrm{k}$ datasets. We then found the average number of false positives and true negatives as well as the number of false negatives and true positives in every pathway for each $\mathrm{k}$. This allowed us to calculate the specificity, sensitivity, precision and negative prediction value. In this context, sensitivity thus quantifies the prioritisation approach's ability to correctly prioritise the "defector gene". Similarly, specificity allows judging an approach's tendency to prioritise random genes that are not involved in the disease. Generally, a prioritisation approach with high specificity is preferred, as this reduces costs involved in the follow-up of false candidate genes.

Our prioritisation approach (at 20\% correlation threshold) has mean specificity above 0.70 for any one of the brain array resources, or combinations thereof, for both the KEGG and PsyGeNet set of known genes (compare Figs. 1 and 2). Sensitivity rapidly decreases with the number of datasets required to prioritise the "defector" genes. Requiring a gene to be prioritised in at least two datasets seems to result in the best trade-off between specificity and sensitivity of the method when precision and negative prediction value (Additional file 1: Figures S8 and S9) are also considered. It is interesting to note that the sensitivity values for the PsyGeNet sets are only slightly below those found for the KEGG gene sets. This suggests that gene expression networks can be utilized for the identification of disease genes in psychiatric diseases much like for the construction of pathways. A large gene co-expression network functionally related to synaptic transmission and recently identified to be differentially regulated in schizophrenia is further testament to this [38]. It also suggests the utility of brain-coX for the interpretation of neuropsychiatric GWAS results.

\section{Benchmarking of different cleaning strategies}

We also compared prioritisation accuracy for the two different normalisation techniques implemented in brain-coX, RUV and background correction combined with quantile normalisation. We determined sensitivity and specificity from leave-one-out cross-validation on the 37 KEGG pathways as described previously. Using these pathways, sensitivity appears to be roughly similar for the two approaches (Additional file 1: Figure $\mathrm{S} 10)$. There are statistically significant gains in specificity $(t$-test, $t$-statistics $=8.49, p$ value $=7.73 \mathrm{e}-12)$ when using RUV normalisation compared to conventional normalisation with background-correction and quantile normalisation. Our previous work published on RUV normalisation applied to correlations indicated greater reproducibility of prioritisation results between datasets for epileptic encephalopathy candidates when using the RUV approach compared to the conventional approach [28].

\section{Comparison with other web-applications}

Comparing the performance of brain-coX with other prioritisation web-tools is challenging. Most web-based prioritisation tools already integrate databases such as OMIM, DisGeNet [39] and KEGG in order to improve their performance. Thus, neither the KEGG pathways
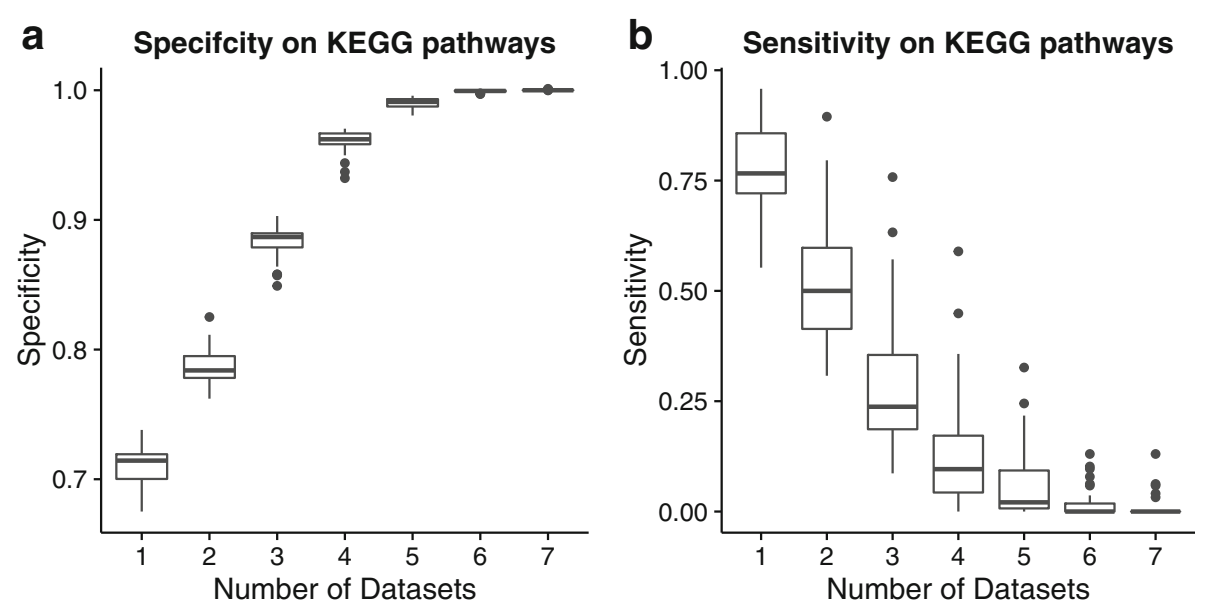

Fig. 1 Accuracy of brain-coX in predicting KEGG pathways. The displayed accuracy measures were generated from leave-one-out cross-validation using 37 KEGG pathways that function in the human brain. We also examine the effect of requiring a gene to be prioritised in multiple datasets on the accuracy measures. a Specificity of brain-coX prioritisation approach. $\mathbf{b}$ Sensitivity of the brain-coX prioritisation approach 

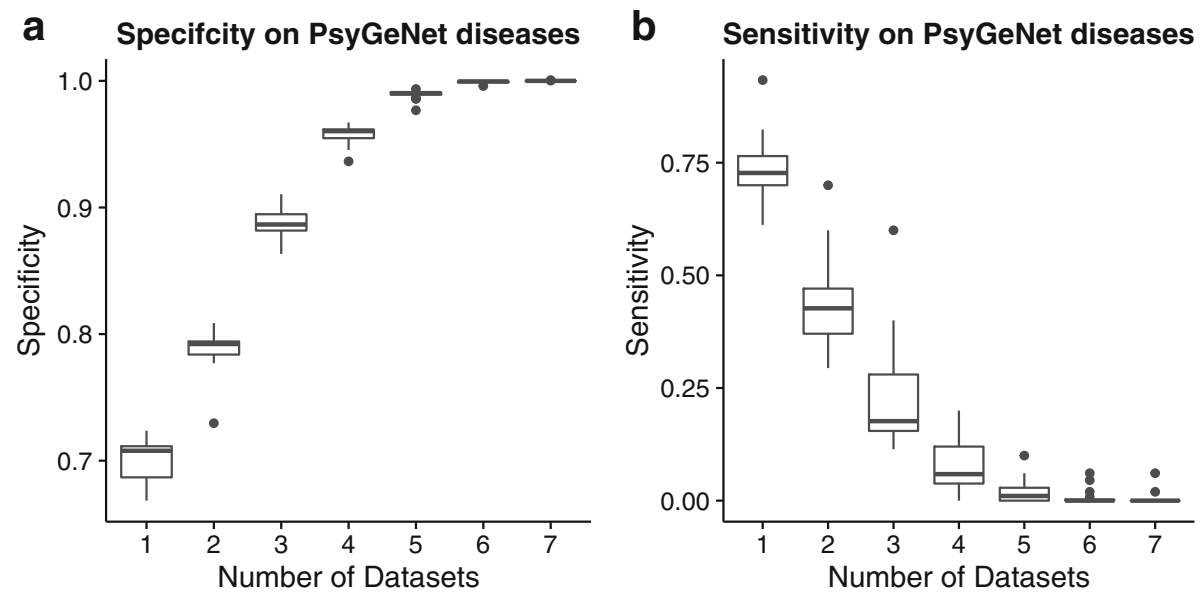

Fig. 2 Accuracy of brain-coX in predicting disease genes in PsyGeNet. The displayed accuracy measures were generated from leave-one-out cross-validation using 17 PsyGeNet diseases that function in the human brain. We also examine the effect of requiring a gene to be prioritised in multiple datasets on the accuracy measures. a Specificity of brain-coX prioritisation approach. $\mathbf{b}$ Sensitivity of the brain-coX prioritisation approach

nor the PsyGeNet disease genes should be used to compare performance of different tools, as such a comparison would be heavily biased in favour of web-tools that make use of these resources. Moreover, such a comparison does not reflect the real user case where these tools are being used to discover novel disease genes and pathways.

We compare brain-coX to two competing web-based prioritisation approaches, Endeavour and ToppGene. Both approaches rank candidate genes with the help of known disease genes. We chose not to compare the performance of brain-coX to prioritisation approaches that do not offer web interfaces. We acknowledge that such tools could potentially offer superior performance, but they cannot be used without some knowledge of programming. One example is Weighted Gene CoExpression Network Analysis (WGCNA) [14], the most commonly used network construction tool. While this tool requires extensive optimization of parameters, it allows temporal effects to be taken into account. We did investigate how the performance of WGCNA compares to our approach for a subset of 37 KEGG pathways described above. We found that brain-coX performs better, distinguishing between genuine pathway genes and random genes, than WGCNA (Additional file 1). Note that we also do not compare brain-coX to prioritisation tools specialised for only one disease, such as the algorithm Detecting Association With Network (DAWN) for autism [6], which uses expert knowledge. Such tools might outperform brain-coX. However, for most neurological and mental diseases specialised tools do not yet exist.

In order to overcome the bias in the leave-one-out cross-validation studies with known pathways, we also investigated a set of genes that a priori is expected to harbour several true positive genes. We made use of the curated Simons Foundation Autism Research Initiative (SFARI) Gene database [40], which is expected to contain likely disease-causing autism genes. These genes are furthermore ranked in terms of confidence, providing a further source of data for validation. The 826 genes in the database are scored from 1 to 6 ; each value indicating a category. The first category includes highconfidence autism genes and the last category contains genes that are currently not supported by any evidence for their involvement in autism.

To compare the performance of Endeavour, ToppGene and brain-coX, we used the 17 genes in the highconfidence group (category 1) as our known autism genes. We designated 340 genes in categories 2 to 6 that were not associated with autism in DisGeNet as candidate autism genes. We restricted brain-coX to only use samples collected from early mid-fetal development to early childhood (periods 4-10). Autism typically manifests in the first or second year of life [41], but there is robust evidence for the involvement of early mid-fetal development in this disease [42]. We used both ToppGene and Endeavour largely with default settings; however, we excluded the use of BLAST Annotation for Endeavour to ensure completion of the prioritisation with large sets of candidates. brain-coX prioritised 222 genes at $20 \%$ threshold in at least one dataset, while ToppGene prioritised all candidates and Endeavour prioritised only two genes according to the associated $p$ values.

In order to assess the quality of the prioritisation of each approach we examined the cumulative average of the gene score assigned by SFARI Gene to the first 100 ranked genes (compare Fig. 3). Thus, a low score indicates good performance. The cumulative average score for the genes prioritised by brain-coX was either equal 


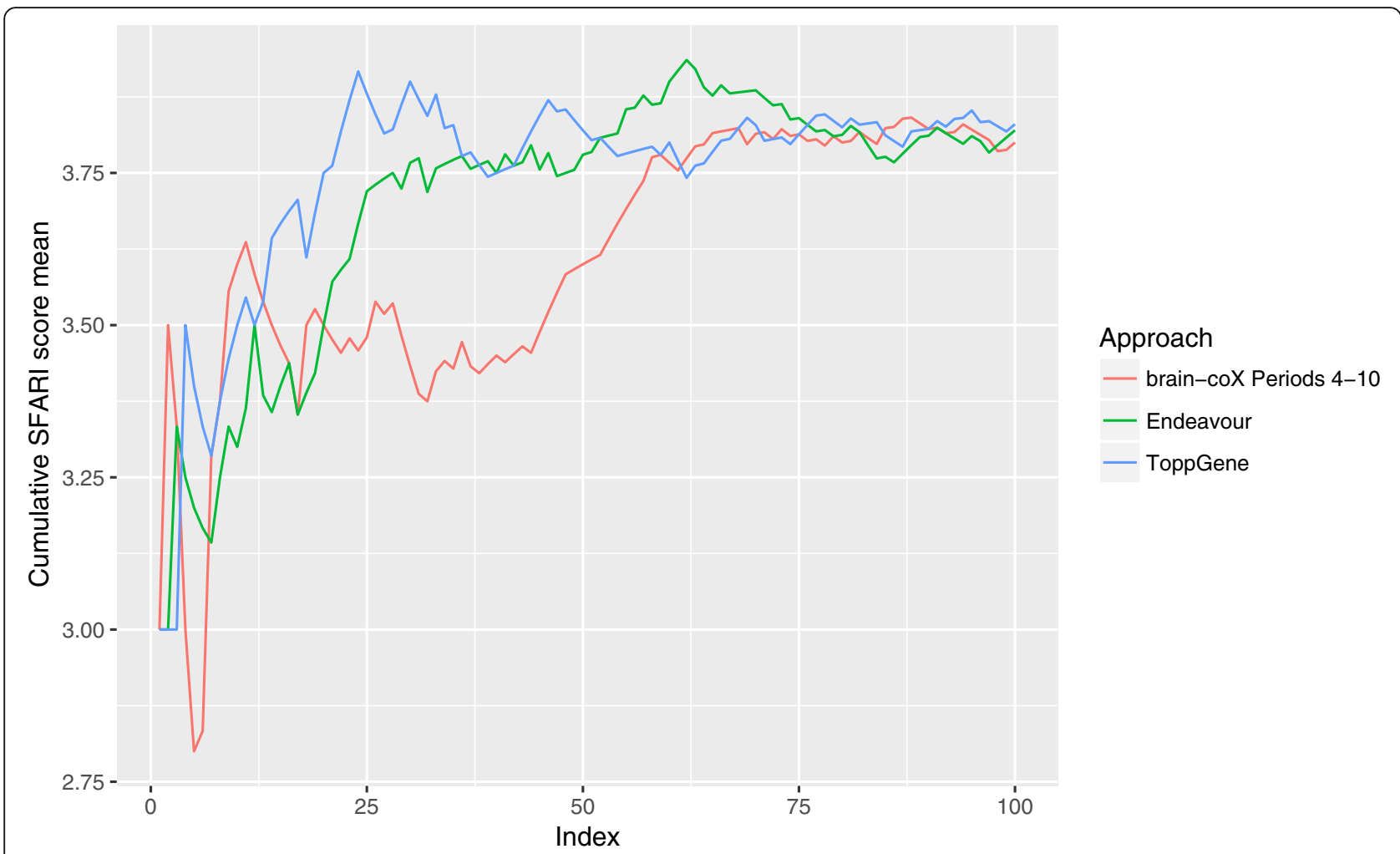

Fig. 3 Cumulative mean score for SAFRI candidate genes. We prioritised 340 genes in the SAFRI database for autism with three different prioritisation approaches given 17 known autism genes. For the first 100 prioritised genes of each method we calculated the cumulative mean of the respective SFARI scores (2-6). Lower scores indicate genes that are more likely to be involved in autism

to or lower than that with either Endeavour or ToppGene. While there was not much difference between the different prioritisation approaches for the first 20 genes, brain-coX yielded considerably lower scores for genes ranked from 21 to 59 . This indicates that brain-coX prioritises genes at least until rank 50 that have more support for involvement in autism than the other tools.

The SFARI Gene database relies on the research community for the collection of autism genes and is thus expected to be incomplete. Furthermore, it is governed by its own set of annotation rules, which may create certain biases. Because of this, we conducted a second performance analysis which was identical to the first in all but the choice of candidate genes. Here, the candidate genes were chosen from an association analysis conducted by Sanders et al. [43] on data from the Autism Genome Project, the Autism Sequencing Consortium and the Simons Simplex Collection. In total, we used 41 genes that reached significance (false discovery rate (FDR) $\leq 0.1$ ) for association with autism and were not found in DisGeNet.

To compare the approaches we examined the Spearman correlation between the prioritisation rank and the FDR value of association with autism for all prioritised genes. Given the wealth of data used by Sanders et al., the FDR value can be viewed as a proxy of the likelihood of true involvement in autism. The Spearman correlation for brain-coX was the highest at 0.259 (0.135 for Endeavour, 0.165 for ToppGene).

\section{Conclusions}

brain-coX will help researchers explore candidate genes and their potential involvement in mental or neurological disorders via in silico prioritisation methods as well as allowing novel visualisation approaches. Thus, brain-coX is the ideal first step in the discovery of novel biomarkers for brain disorders or the development of new treatments for such illnesses. It is important to remember that any candidate genes prioritised by braincoX need to be followed up to confirm their suspected involvement in the investigated brain disorder. Followup usually includes multiple different experimental as well as observational avenues, including, but not limited to, animal models and human iPSCs studies.

brain-coX is underpinned by the world's largest resource of human brain microarray datasets ever assembled. By exclusively focusing on gene expression measurements in post-mortem human brain, we created a tool that is not biased by what is already known from 
literature or experimental approaches, but targeted to diseases originating in the brain. brain-coX also allows insights into the temporal complexity of the human brain within an easy to use web-tool. This means that brain-coX is uniquely suited towards improving our understanding of normal regulation throughout brain development. Unfortunately our effort to also make use of brain regions was thwarted due to the large inconsistencies in brain anatomical annotation between the different datasets and remains a future research goal and extension for brain-coX.

\section{Availability and requirements}

brain-coX is available via http://shiny.bioinf.wehi.edu.au/ freytag.s/. A stand-alone version is available upon request, but requires $\mathrm{R}$.

\section{Additional files}

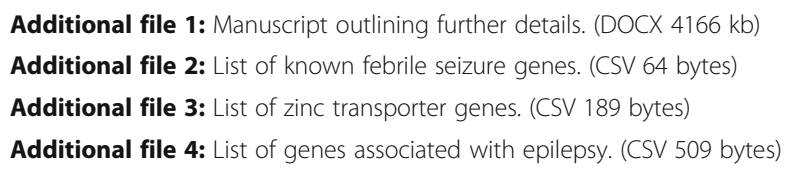

\section{Acknowledgements}

We would like to acknowledge Adam O'Neill, Lutz Freytag and Peter Hickey for help with testing the software and making valuable suggestions for further improvement. We would also like to thank the reviewers whose suggestions and corrections improved not only the manuscript but also the tool itself.

\section{Funding}

This work was supported by the Victorian Government's Operational Infrastructure Support Program and Australian Government NHMRC IRIIS. MB is funded by NHMRC Senior Research Fellowship 110297 and NHMRC Program Grant 1054618.

\section{Availability of data and materials \\ brain-coX can be freely accessed via http://shiny.bioinf.wehi.edu.au/freytag.s/. The datasets supporting the conclusions of this article are available in the Allen Institute for Brain Science repository (http://human.brain-map.org/ static/download), in the BrainSpan repository (http://www.brainspan.org/ static/download.html and http://download.alleninstitute.org/brainspan/ MRF_BigWig_Gencode_v10/bigwig/) and NCBI's Gene Expression Omnibus (accession numbers GSE30272, GSE36192 and GSE60862).}

\section{Authors' contributions}

SF prepared the manuscript, programmed the software and conducted the analysis. $\mathrm{MB}$ contributed ideas for the software and contributed to the interpretation of all results. KLO and RB contributed towards the interpretation of results regarding febrile seizures and autism. All authors were involved in the editing of the manuscript. All authors read and approved the final manuscript.

\section{Competing interests}

The authors declare that they have no competing interests.

\section{Consent for publication}

Not applicable.

Ethics approval and consent to participate Not applicable.

\section{Publisher's Note}

Springer Nature remains neutral with regard to jurisdictional claims in published maps and institutional affiliations.

\section{Author details}

'Population Health and Immunity Divison, The Walter and Eliza Hall Institute of Medical Research, 1G Royale Parade, 3052 Parkville, Australia. ${ }^{2}$ Department of Medical Biology, University of Melbourne, 1G Royale Parade, 3052 Parkville, Australia. ${ }^{3}$ Epilepsy Research Centre, Department of Medicine, Austin Health, University of Melbourne, 245 Burgundy Street, 3084 Heidelberg, Australia. ${ }^{4}$ School of Mathematics and Statistics, University of Melbourne, 3010 Parkville, Australia.

Received: 21 December 2016 Accepted: 26 May 2017

Published online: 08 June 2017

\section{References}

1. World Health Organization. Mental health action plan 2013-2020. Geneva: World Health Organization; 2013.

2. Simmons JM, Quinn KJ. The NIMH Research Domain Criteria (RDoC) Project: implications for genetics research. Mamm Genome. 2013;25(1-2):23-31.

3. Gatt JM, Burton KLO, Williams LM, Schofield PR. Specific and common genes implicated across major mental disorders: A review of meta-analysis studies. J Psychiatr Res. 2015;60:1-13.

4. Hu WF, Chahrour MH, Walsh CA. The diverse genetic landscape of neurodevelopmental disorders. Annu Rev Genomics Hum Genet. 2014;15: 195-213.

5. Sekar A, Bialas AR, de Rivera H, Davis A, Hammond TR. Kamitaki, et al. Schizophrenia risk from complex variation of complement component 4. Nature. 2016;530(7589):177-83.

6. Liu L, Lei J, Sanders SJ, Willsey AJ, Kou Y, Cicek AE, et al. DAWN: A framework to identify autism genes and subnetworks using gene expression and genetics. Mol Autism. 2014;5(1):1

7. Amberger JS, Bocchini CA, Schiettecatte F, Scott AF, Hamosh A. OMIM.org: Online Mendelian Inheritance in Man (OMIM ${ }^{\circ}$ ), an online catalog of human genes and genetic disorders. Nucleic Acids Res. 2015;43(Database Issue):789-98.

8. Moreau Y, Tranchevent L-C. Computational tools for prioritizing candidate genes: boosting disease gene discovery. Nat Rev Genet. 2012;13(8):523-36.

9. Tiffin N, Andrade-Navarro MA, Perez-Iratxeta C. Linking genes to diseases: it's all in the data. Genome Med. 2009;1(8):1-7.

10. Aerts S, Lambrechts D, Maity S, Van Loo P, Coessens B, De Smet F, et al. Gene prioritization through genomic data fusion. Nat Biotechnol. 2006; 24(5):537-44.

11. Chen J, Bardes EE, Aronow BJ, Jegga AG. Toppgene suite for gene list enrichment analysis and candidate gene prioritization. Nucleic Acids Res. 2009:37 suppl 2:305-11.

12. Szklarczyk D, Franceschini A, Kuhn M, Simonovic M, Roth A, Minguez P, et al. The string database in 2011: functional interaction networks of proteins, globally integrated and scored. Nucleic Acids Res. 2011;39 Suppl 1:561-8.

13. Piro RM, Di Cunto F. Computational approaches to disease-gene prediction: rationale, classification and successes. FEBS J. 2012;279(5):678-96.

14. Langfelder P, Horvath S. WGCNA: an R package for weighted correlation network analysis. BMC Bioinf. 2008;9(1):1.

15. PubMed by NCBI. https://www.ncbi.nlm.nih.gov/pubmed. Accessed 12 Sept 2015.

16. Antanaviciute A, Daly C, Crinnion LA, Markham AF, Watson CM, Bonthron DT, Carr IM. GeneTIER: Prioritization of candidate disease genes using tissuespecific gene expression profiles. Bioinformatics. 2015;31(16):2728-35.

17. Gaiteri C, Ding Y, French B, Tseng GC, Sibille E. Beyond modules and hubs: the potential of gene coexpression networks for investigating molecular mechanisms of complex brain disorders. Genes Brain Behav. 2013;13(1):13-24.

18. GTEx Consortium. The genotype-tissue expression (GTEx) pilot analysis: multitissue gene regulation in humans. Science. 2015;348(6235):648-60.

19. Oliver KL, Lukic V, Freytag S, Scheffer IE, Berkovic SF, Bahlo M. In silico prioritization based on coexpression can aid epileptic encephalopathy gene discovery. Neurol Genet. 2016;2(1):51.

20. Kang HJ, Kawasawa Yl, Cheng F, Zhu Y, Xu X, Li M, et al. Spatio-temporal transcriptome of the human brain. Nature. 2011;478(7370):483-9.

21. Gilman SR, lossifov I, Levy D, Ronemus M, Wigler M, Vitkup D. Rare de novo variants associated with autism implicate a large functional network of genes involved in formation and function of synapses. Neuron. 2011;70(5): 898-907. 
22. Hernandez DG, Nalls MA, Moore M, Chong S, Dillman A, Trabzuni D, et al. Integration of GWAS SNPs and tissue specific expression profiling reveal discrete eQTLs for human traits in blood and brain. Neurobiol Dis. 2012;47(1):20-8.

23. Johnson WE, Li C, Rabinovic A. Adjusting batch effects in microarray expression data using empirical Bayes methods. Biostatistics. 2007:8(1):118-27.

24. Irizarry RA, Hobbs B, Collin F, Beazer-Barclay YD, Antonellis KJ, Scherf $\mathrm{U}$, Speed TP. Exploration, normalization, and summaries of high density oligonucleotide array probe level data. Biostatistics. 2003;4(2):249-64.

25. Bolstad BM, Irizarry RA, Åstrand M, Speed TP. A comparison of normalization methods for high density oligonucleotide array data based on variance and bias. Bioinformatics. 2003;19(2):185-93.

26. Gagnon-Bartsch JA, Speed TP. Using control genes to correct for unwanted variation in microarray data. Biostatistics. 2012;13(3):539-52.

27. Walker WL, Liao IH, Gilbert DL, Wong B, Pollard KS, McCulloch CE, Lit L, Sharp FR. Empirical Bayes accommodation of batch-effects in microarray data using identical replicate reference samples: application to RNA expression profiling of blood from Duchenne muscular dystrophy patients. BMC Genomics. 2008;9(1):1.

28. Freytag S, Gagnon-Bartsch J, Speed TP, Bahlo M. Systematic noise degrades gene co-expression signals but can be corrected. BMC Bioinf. 2015;16(1):462.

29. Kolaczyk ED, Csárdi G. Statistical analysis of network data with R. 1st ed. New York: Springer; 2014.

30. Oliver KL, Lukic V, Thorne NP, Berkovic SF, Scheffer IE, Bahlo M. Harnessing gene expression networks to prioritize candidate epileptic encephalopathy genes. PLoS One. 2014;9(7):102079.

31. Hawrylycz MJ, Lein ES, Guillozet-Bongaarts AL, Shen EH, Ng L, Miller JA, et al. An anatomically comprehensive atlas of the adult human brain transcriptome. Nature. 2012;489(7416):391-9.

32. Rubin TG, Gray JD, McEwen BS. Experience and the ever-changing brain: What the transcriptome can reveal. Bioessays. 2014;36(11):1072-81.

33. Shiny by RStudio. http://shiny.rstudio.com. Accessed 22 Nov 2015

34. R: The R Project For Statistical Computing. https://www.r-project.org. Accessed 22 Nov 2015.

35. Bioconductor Open Source Software for Bioinformatics. https://www. bioconductor.org. Accessed 22 Nov 2015

36. Kanehisa M, Sato Y, Kawashima M, Furumichi M, Tanabe M. KEGG as a reference resource for gene and protein annotation. Nucleic Acids Res. 2016;44(D1):457-62.

37. Gutiérrez-Sacristán A, Grosdidier S, Valverde O, Torrens M, Bravo A, Piñero J, et al. PsyGeNet: a knowledge platform on psychiatric disorders and their genes. Bioinformatics. 2015;31:3075-7.

38. Hertzberg L, Katsel P, Roussos P, Haroutunian V, Domany E. Integration of gene expression and GWAS results supports involvement of calcium signaling in schizophrenia. Schizophr Res. 2015;164(1):92-9.

39. Piñero J, Queralt-Rosinach N, Bravo À, Deu-Pons J, Bauer-Mehren A, Baron $M$, et al. DisGeNet: A discovery platform for the dynamical exploration of human diseases and their genes. Database. 2015;2015:bav028.

40. Banerjee-Basu S, Packer A. SFARI gene: an evolving database for the autism research community. Dis Model Mech. 2010;3(3-4):133-5.

41. Ozonff S, Heung K, Byrd R, Hansen R, Hertz-Picciotto I. The onset of autism: patterns of symptom emergence in the first years of life. Autism Res. 2008; 1(6):320-8.

42. Willsey AJ, Sanders SJ, Li M, Dong S, Tebbenkamp AT, Muhle RA, Reilly SK, et al. Coexpression networks implicate human midfetal deep cortical projection neurons in the pathogenesis of autism. Cell. 2013;155(5):997-1007.

43. Sanders SJ, He X, Willsey AJ, Ercan-Sencicek AG, Samocha KE, Cicek AE, et al. Insights into autism spectrum disorder genomic architecture and biology from 71 risk loci. Neuron. 2015;87(6):1215-33.

44. Miller JA, Ding S-L, Sunkin SM, Smith KA, Ng L, Szafer A, et al. Transcriptional landscape of the prenatal human brain. Nature. 2014; 508(7495):199-206.

45. Colantuoni C, Lipska BK, Ye T, Hyde TM, Tao R, Leek JT, et al. Temporal dynamics and genetic control of transcription in the human prefrontal cortex. Nature. 2012;478(7370):519-23.

46. Trabzuni D, Ramasamy A, Imran S, Walker R, Smith C, Weale, et al. North American Brain Expression Consortium: Widespread sex differences in gene expression and splicing in the adult human brain. Nat Commun. 2013;4:2771.

47. Zhang B, Gaiteri C, Bodea L-G, Wang Z, McElwee J, Podtelezhnikov AA Zhang, et al. Integrated systems approach identifies genetic nodes and networks in late-onset Alzheimer's disease. Cell. 2013;153(3):707-20.

\section{Submit your next manuscript to BioMed Central and we will help you at every step:}

- We accept pre-submission inquiries

- Our selector tool helps you to find the most relevant journal

- We provide round the clock customer support

- Convenient online submission

- Thorough peer review

- Inclusion in PubMed and all major indexing services

- Maximum visibility for your research

Submit your manuscript at www.biomedcentral.com/submit 